\title{
The FedEx Problem
}

\author{
AMOS FIAT, Tel Aviv University \\ KIRA GOLDNER, University of Washington \\ ANNA R. KARLIN, University of Washington \\ ELIAS KOUTSOUPIAS, University of Oxford
}

\section{CCS Concepts: •Theory of computation $\rightarrow$ Algorithmic mechanism design; Computational pricing and auctions;}

Additional Key Words and Phrases: Mechanism design; optimal auctions; price discrimination

"Remember that Time is Money"

- Benjamin Franklin in Advice to a Young Tradesman (1748)

Consider the pricing problem faced by FedEx. Each customer has a package to ship, a deadline $d$ by which he needs his package to arrive, and a value $v$ for a guarantee that the package will arrive by his deadline. FedEx can (and does) offer a number of different shipping options in order to extract more revenue from their customers. In this paper, we solve the optimal (revenue-maximizing) auction problem for the singleagent version of this problem. Our paper adds to the relatively short list of multiparameter settings for which a closed-form solution is known.

This pricing problem is extremely natural and arises in numerous scenarios, whether it is Amazon.com providing shipping options, Internet Service Providers offering bandwidth plans, Bitcoin miners setting a policy for transaction fees, or a myriad of other settings in which customers have a sensitivity to time or some other feature of service. In these settings, a seller can price discriminate or otherwise segment his market by delaying service, or providing lower quality/cheaper versions of a product. It is important to understand how buyer deadline (or quality) constraints impact the design of auctions and what leverage they give to the seller to extract more revenue.

We consider a model in which a seller provides $n$ different options for service, and a customer is interested in buying an option that meets his quality demand of $d$. We use the running example of shipping packages by deadlines. A customer's utility for getting his package shipped by day $t$ at a price of $p$ is $v-p$ if $t \leq d$ (i.e., it is received by his deadline) and $-p$ otherwise. A customer's $(v, d)$ pair is his private information. We study the Bayesian setting, where this pair $(v, d)$ is drawn from a prior distribution known to the seller.

This research was done in part while the authors were visiting the Simons Institute for Theoretical Computer Science.

The second and third authors are funded by the National Science Foundation under CCF grant 1420381. The fourth author is funded by the European Research Council under the European Union's Seventh Framework Programme (FP7/2007-2013) / ERC grant agreement no. 321171 (ALGAME). Authors addresses:

A. Fiat, School of Computer Science, Tel Aviv University; email: fiat@tau .ac.il

K. Goldner and A.R. Karlin, Department of Computer Science and Engineering, University of Washington; email: $\{$ kgoldner, karlin $\}$ @cs . washington.edu;

E. Koutsoupias, Department of Computer Science, University of Oxford; email: elias@cs.ox.ac.uk

Permission to make digital or hard copies of part or all of this work for personal or classroom use is granted without fee provided that copies are not made or distributed for profit or commercial advantage, and that copies bear this notice and the full citation on the first page. Copyrights for third-party components of this work must be honored. For all other uses, contact the owner/author(s). Copyright is held by the author/owner(s).

EC'16, July 24-28, 2016, Maastricht, The Netherlands.

ACM 978-1-4503-3936-0/16/07.

http://dx.doi.org/10.1145/2940716.2940752 
Our main result is a characterization of the revenue-optimal auction for this setting. For each delivery option of 1 through $n$ days, the mechanism specifies a distribution of prices. The customer, knowing the distributions, specifies a delivery option of $i$ days, and then a price is drawn randomly from day $i$ 's distribution and offered to the customer.

We formulate the optimal auction problem as a continuous infinite linear program, take its dual, and determine a sufficient set of conditions for optimality. We then show how to construct a sequence of "revenue-type" curves $\Gamma_{\geq i}(\cdot)$. Each such curve represents the optimal revenue on days $i$ through $n$ given a price that might be set on day $i$. That is, $\Gamma_{\geq i}(p)$ corresponds to the sum of (1) the revenue from selling day $i$ delivery at a price of $p$ to customers with a deadline of $i$ and (2) the optimal revenue (when constrained by this choice on day $i$ ) from days $i+1$ through $n$, where the constraint comes from the requirement that it is incentive compatible for a customer to report his true deadline rather than an earlier one. This curve also incorporates "ironing" so as to ensure incentive compatibility, and this ironing may lead to randomization over prices. We use these curves to construct a solution to the primal and dual linear programs that satisfies conditions sufficient for optimality.

Our result is one of relatively few exact and explicit closed-form generalizations of [Myerson 1981] to multi-parameter settings with arbitrary joint distributions, and contributes to recent breakthroughs in this space. Key take-aways are the following:

(1) Our result requires a new way of combining and ironing revenue curves. In Myerson's optimal auction for irregular distributions, ironing ensures incentive compatibility and gives an upper bound on the optimal revenue. Myerson shows that this upper bound is in fact achievable using randomization. Similarly, our combined and ironed curves yield upper bounds on the revenue, and we show that these upper bounds can be realized with lotteries. In Myerson's setting, ironing is required to enforce incentive compatibility constraints among multiple bidders. In our setting, we need ironing even for one bidder because of the multiple parameters. This may suggest that ironing is one of the biggest hurdles in extending Myerson's results to more general settings.

(2) The optimal auction we obtain is constructed inductively and, consequently, is relatively simple to describe. Once the distribution of prices has been determined for delivery by day $i$, using $\Gamma_{\geq i+1}(\cdot)$, we show how to define the distribution of prices for delivery by day $i+1$. The latter involves randomizing over up to $2^{i}$ prices.

(3) The duality approach gives a closed-form allocation rule. Naive attempts to solve this problem, even for the case where there are only two or three possible deadlines, leads to a massive case analysis depending on the priors. Even in cases where the optimal auction is deterministic, setting these prices is not straightforward. For example, for three possible deadlines, the optimal deterministic mechanism can require 1,2 , or 3 distinct prices, and determining how many prices to use and how to set them seems non-trivial.

Our duality approach, however, leads to a unified allocation rule with no case analysis at all. This paper strengthens the emerging understanding that duality is useful for determining the structure of the optimal auction in non-trivial settings and for analyzing the resulting auction.

The full (working) paper, including references to related work, can be found here.

\section{REFERENCES}

Roger B. Myerson. 1981. Optimal Auction Design. Mathematics of Operations Research 6, 1 (1981), 58-73. http://dx.doi.org/10.1287/moor.6.1.58 